\title{
EFFICACY OF ORAL AMOXICILLIN VERSUS PARENTERAL CEFTRIAXONE IN TREATMENT OF UNCOMPLICATED COMMUNITY ACQUIRED PNEUMONIA (CAP): A PROSPECTIVE, SINGLE BLINDED, PARALLEL DESIGN, RANDOMIZED CONTROLLED TRIAL
}

\author{
Sushant S Mane, Vaijnath Sathe, Manas V Pustake, Sagar Walhekar, Sharanya Ramakrishnan. \\ Department of Pediatrics, Grant Government Medical College and Sir J.J. Group of Hospitals, Byculla, \\ Mumbai, 400008, India.
}

\begin{abstract}
Aim: Community acquired Pneumonia (CAP) is one of the major causes of under-five morbidity and mortality in most of the tropical countries. Management of CAP with an appropriate antibiotic will help reduce the growing antibiotic resistance. The aim of the study was to compare the efficacy of oral amoxicillin with parenteral ceftriaxone in uncomplicated CAP in children aged 6 months to 12 years.
\end{abstract}

Methods: This was a prospective, single blinded, parallel design, randomized control trial. One hundred children with uncomplicated CAP, aged 6 months to 12 years, admitted in pediatric wards at a tertiary care centre were included in the study. The enrolled patients were randomized in two groups in the ratio of $1: 1$. Group I received oral amoxicillin and group II received intravenous ceftriaxone for 7 days. Number of days for defervescence, day of settlement of respiratory distress, day of subsidence of cough, duration of hospital stay, step-up to higher antibiotics, adverse drug reactions were compared in both the groups.

Results: Time to deferervescence of fever in Group I was 3.7 \pm 0.9 days as compared to $3.66 \pm 0.8$ days in group II $(p=0.81)$. Settlement of respiratory distress occurred in $3.22 \pm 0.85$ days in group I as compared to $3 \pm 0.67$ days in group II $(p=0.19)$. Subsidence of cough occurred in $11.68 \pm 0.92$ days in group I as compared to $11.14 \pm 1.06$ days in group II $(p=0.74)$. Duration of hospital stay was $6.02 \pm 1.11$ days in group I as compared to $7.24 \pm 1.18$ days in group II $(p<0.0001)$. Step-up to a higher antibiotic was required in $2(4 \%)$ children on oral amoxicillin and $1(2 \%)$ child on IV. ceftriaxone $(p=1.00)$. Two children from oral amoxicillin group had diarrhea without any signs of dehydration on day 2 of treatment, which subsided in 2 days after giving probiotics and oral rehydration solution. This did not require discontinuation of amoxicillin. No side effects were observed from IV ceftriaxone group.

Conclusion: Use of oral amoxicillin for uncomplicated CAP in children has a similar outcome as compared to parenteral cephalosporins. It reduces the duration of hospital stay and thus the cost of treatment. More importantly, it prevents the unnecessary use of a higher-class antibiotic, thereby helping to reduce antibiotic resistance.

\section{Introduction}

Pneumnia remains the single largest infectious cause of death in children worldwide. ${ }^{1}$ Globally, more than 800,000 children die of pneumonia annually before their fifth birthday. ${ }^{2}$ Incidence of pneumonia in low and middle-income countries (LMIC) is ten times that of high-income countries (HIC), which is attributable to a low birth weight, malnutrition, vitamin A deficiency, lack of breastfeeding, passive smoking, poor socioeconomic

Address for Correspondence: Manas V Pustake Room 230, Apna Boys' Hostel, JJ Hospital Campus, Byculla, Mumbai 400008, India.

Email: pustakemanas@gmail.com

(C)2021 Pediatric Oncall
ARTICLE HISTORY

Received 30 December 2020

Accepted 4 March 2021

\section{KEYWORDS}

Community acquired pneumonia, amoxicillin, cephalosporin, randomized control trial, uncomplicated pneumonia status, large family size, overcrowding and pollution in these countries. ${ }^{3}$ Nevertheless, community acquired pneumonia (CAP) incurs huge costs either directly for treatment or through loss of working hours by guardians of diseased children. ${ }^{4}$

CAP is an infection of lung parenchyma in a previously healthy patient who acquired the infection in the community and has no history of hospitalization in the 2 weeks preceding manifestation. ${ }^{5,6}$ The most common causes of uncomplicated CAP are Hemophilus influenzae, Streptococcus pneumoniae, Staphylococcus aureus, certain gram-negative rods, certain viruses and atypical organisms. ${ }^{6,7,8}$ The clinical diagnosis of CAP is made using the new World Health Organization (WHO) criteria5 which primarily includes two categories 
of pneumonia: 1) pneumonia with fast breathing and/ or chest indrawing, which requires home therapy with oral amoxicillin, and 2) severe pneumonia defined as pneumonia with any general danger sign (including inability to drink/feed, persistent vomiting, convulsions, lethargy or unconsciousness, stridor in a calm child or severe malnutrition). Uncomplicated CAP is defined as CAP in which there are no signs of other complications such as pleural effusion, cavitation or abscess from admission to discharge. ${ }^{9}$

A microbial culture takes significant amount of time. This could potentially delay initiation of treatment. Without microbiological culture, we cannot accurately predict the causative agent. This justifies the role of empirical treatment with higher antibiotics in CAP. ${ }^{10}$ The current management guidelines focus more on diagnostic sensitivity than specificity, leading to abuse of higher antibiotics. ${ }^{11,12}$ WHO recommends first line treatment with twice daily amoxicillin given for 7 days, for pneumonia with rapid breathing (age appropriate measure) with or without chest indrawing but no danger signs, in children under 5 years of age. ${ }^{5}$ While there is no standard recommendation of IV ceftriaxone for 7 days for an uncomplicated CAP, it is being used rampantly in clinical settings for the treatment of uncomplicated CAP. ${ }^{13,14,15,16}$ Also, many studies from LMICs suggest the use of ceftriaxone for severe pneumonia. 17,18,19,20,21,22 However, some studies suggest that the use of narrow-spectrum antibiotics against CAP is as useful as broad-spectrum antibiotics. ${ }^{22}$ If proven equally efficacious, the use of lower generation antibiotics with high therapeutic indices, like penicillin or amoxicillin must definitely be made a priority. In addition to contributing to drug resistance, adverse reactions to cephalosporins must also be considered while prescribing these antibiotics. ${ }^{22}$ Additionally, in LMI countries, use of narrow spectrum antibiotics will also be a cost-effective alternative for treating CAP.

The objective of our study was to assess the efficacy of oral amoxicillin versus parenteral cephalosporin in uncomplicated CAP among children 6 months to 12 years of age.

\section{Methods \& Materials}

This study was a prospective, single blinded, parallel design, randomized control trial in a tertiary care hospital of a metropolitan city in Western India conducted over a period of 18 months from January 2018 to August 2019. Children admitted in the pediatric wards were enrolled for the study. Patients were blinded regarding the drug administered throughout the study. One hundred children between 6 months to 12 years of age, diagnosed with uncomplicated CAP (presenting with fast breathing or chest indrawing but no danger signs) were enrolled. 5,9 Children admitted in Pediatric Intensive Care Unit (PICU), those with underlying immunodeficiency disorders (acquired or congenital), with other co-existing infections (for example: dengue, malaria, measles etc.) or with underlying co-morbidities like cystic fibrosis, sickle cell disease, disorders of cilia etc., children with underlying anatomical disorders of the lung like pulmonary sequestration, tracheo-esophageal fistula, congenital cystic adenoid malformation or any other co-existing acute or chronic disorders were excluded from the study. After obtaining approval from the Institutional Ethics Committee (IEC), a written informed consent was taken from the caretakers. The study was registered with the Clinical Trials Registry - India (CTRI) [CTRI/2019/07/020174].

Children presenting with clinical features of CAP viz. tachypnea, respiratory distress, fever, were evaluated using the WHO criteria for clinical diagnosis of pneumonia. ${ }^{5}$ A chest X-ray was done for all and cases having radiological evidence of lobar and/or bronchopneumonia were enrolled. Chief complaints, co-morbidities, family \& socioeconomic history, birth history, and immunization history of the patients were recorded. Physical examination along with baseline investigations like complete blood count, blood culture, sputum for gram stain and culture sensitivity, arterial blood gases ( $A B G$ ) done on the day of admission were recorded. The enrolled patients were divided in two groups in the ratio of $1: 1$. Group I received oral amoxicillin and Group II received intravenous ceftriaxone. Randomization to Group I or II was done by numbering the subjects serially and dividing them into odd and even numbers with odd numbers being included in Group I and even numbers in Group II. After randomization, patients in group I treatment were given oral amoxicillin at $100 \mathrm{mg} / \mathrm{kg} /$ day in three divided doses for 7 days. Patients in group II were given intravenous (IV) ceftriaxone at $75 \mathrm{mg} / \mathrm{kg} /$ day in two divided doses for 7 days. In order to blind the patients from the drug which they were taking, normal saline (NS) intravenously was given and multivitamin syrup orally to all the patients. In this way, all patients were on medications given with both the routes, which made it possible to give the patients drug according to their groups and without revealing the drug. The effectiveness of the two antibiotics were evaluated based on the following parameters: number of days to defervescence of fever (defined as complete abatement of fever for $\geq 24$ hours), days to settlement of respiratory distress, days to subsidence of cough, the duration of hospital-stay, step-up to higher antibiotics, and any significant adverse reactions requiring change of drug. Children from both groups received supplemental oxygen. They were monitored for tachypnea, respiratory distress, fever, cough, oxygen saturations (SpO2) and development of complications. For Group I, children were discharged after the respiratory distress had settled and the children were afebrile for 24 hours. The remaining course of antibiotic was completed at home. Appropriate responses were recorded from these patients' caretakers at the time of discharge on completion of the hospital course. For Group II, children were discharged after 7 days of completion of intravenous antibiotic therapy, provided they were afebrile and respiratory distress had settled. Step-up to higher antibiotics i.e. failure of response, was considered under following circumstances: no improvement and/or worsening of tachypnea, worsening fever or lower chest in-drawing any time before or at 48 hours of admission and/or appearance of danger signs such as inability to drink, abnormal sleepiness, difficult to awake from sleep, stridor in a calm child, central cyanosis, convulsion and/ or occurrence of any complication like empyema, 
pneumothorax, lung abscess, meningitis, septicemia, shock and respiratory failure any time during hospital stay. Children from both groups were followed up at the end of 7 days. Common adverse effects in Group I like diarrhea, vomiting, rashes and in Group II like rash, anaphylaxis, redness and swelling over injection site, diarrhea, if any, were recorded.

\section{Statistical Analysis:}

Data was recorded in Microsoft Excel Version 2016. Descriptive statistics for quantitative variables were represented as mean \pm standard deviation (SD). Qualitative variables were represented as frequency \& percentages. Fisher test or Chi-square test was used to test the association for qualitative/ categorical data. Unpaired sample t-test or Mann Whitney test was used to compare differences between two independent groups depending on the normality of distribution. A p value of $\leq 0.05$ with a confidence interval of $95 \%$ was considered significant.

\section{Results}

One hundred out of 108 eligible children as per the inclusion criteria were recruited. Figure 1 shows the trial profile. Table 1 illustrates baseline characteristics of the participants in both groups.
The minimum age of presentation was 6 months and maximum 11 years. In Group I, 18(36\%) patients were $<1$ year, $25(50 \%)$ were between $1-3$ years, $5(10 \%)$ were between 3-6 years and $2(4 \%)$ patients were $>6$ years. In Group II $21(42 \%)$ patients were $<1$ year, 21(42\%) were between $1-3$ years, $6(12 \%)$ were between 3-6 years and 2(4\%) patients were $>6$ years. Fever was present in all children, as were cough, tachypnea and tachycardia $(n=100)$. The characteristics of fever and cough in both the groups are depicted in table 2.

On blood culture and sputum for gram stain and culture, no organisms were isolated from both groups.

During the treatment, step-up to a higher antibiotic was required in $2(4 \%)$ children on oral amoxicillin and $1(2 \%)$ child on IV. ceftriaxone $(p=1.00)$. Out of two children on oral amoxicillin requiring step-up, one child had respiratory acidosis on admission. The one patient from IV ceftriaxone group who required step-up had respiratory alkalosis on admission. Two children from oral amoxicillin group had diarrhea without any signs of dehydration on day 2 of treatment, which subsided in 2 days after giving probiotics and oral rehydration solution. This did not require discontinuation of amoxicillin. No side effects were observed from

Figure 1. Trial Profile

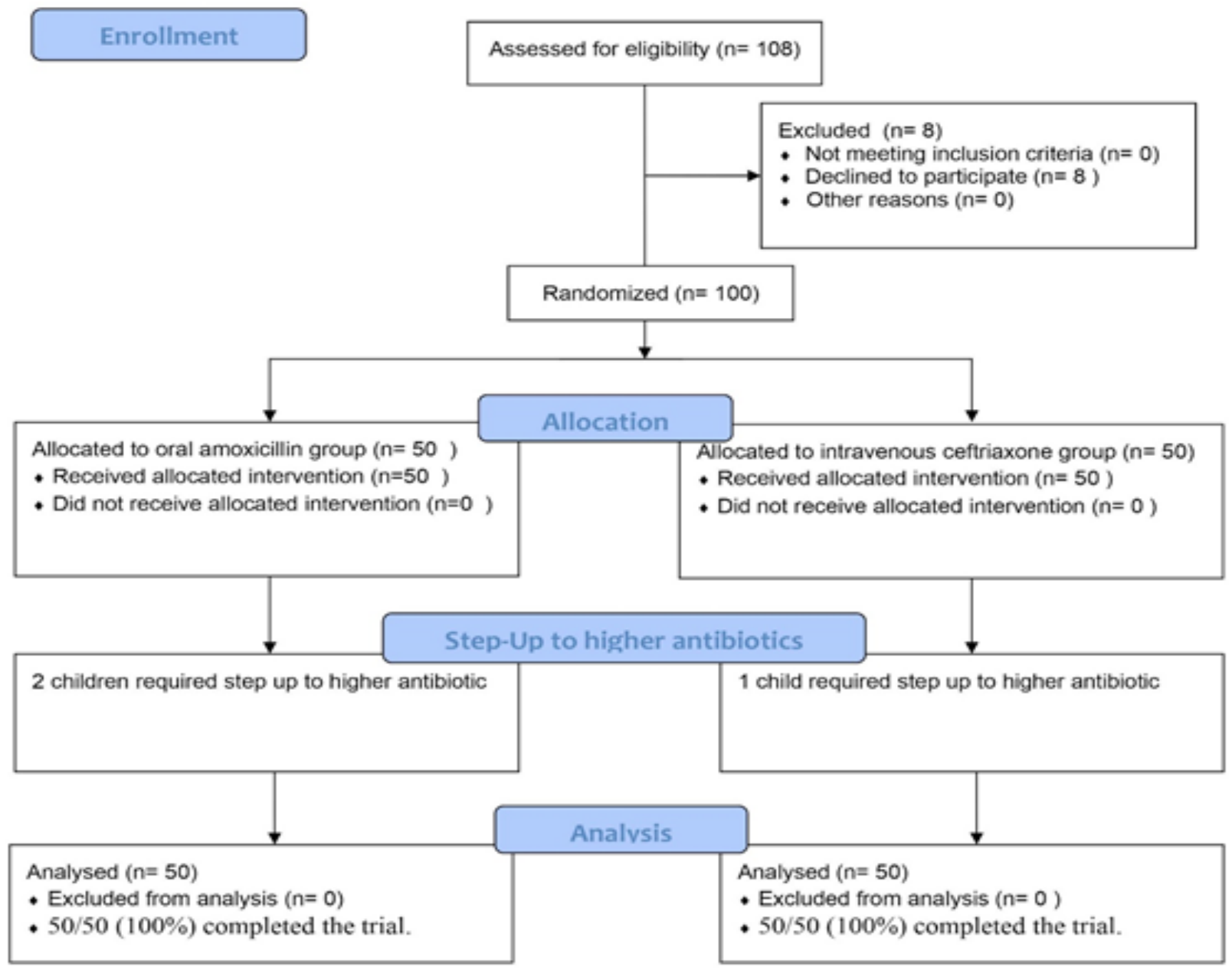


Table 1. Baseline characteristics of all participants

\begin{tabular}{lll}
\hline Baseline Characteristics & Oral amoxicillin group & Intravenous ceftriaxone group \\
\hline Age (Months) & $24.68 \pm 23.70$ & $23.42 \pm 21.31$ \\
Male: Female & $27: 23$ & $22: 28$ \\
Post-Tussive Vomiting & 9 & 2 \\
$\quad$ Vitals \& Complete blood count & & \\
Pulse (/min) & $116.34 \pm 10.58$ & $119.14 \pm 8.23$ \\
Systolic BP (mm of Hg) & $95.48 \pm 5.32$ & $96.45 \pm 4.43$ \\
Diastolic BP (mm of Hg) & $60.02 \pm 4.27$ & $59.82 \pm 4.03$ \\
Respiratory rate (/min) & $52.22 \pm 9.74$ & $54.92 \pm 9.16$ \\
Hemoglobin count (gm/dl) & $9.24 \pm 1.40$ & $9.54 \pm 1.13$ \\
Platelet count (x109/cumm) & $3.42 \pm 1.28$ & $2.88 \pm 1.03$ \\
WBC count (cells/cumm) & $14835 \pm 5932$ & $14720 \pm 5189$ \\
$\quad$ Chest X-Ray Findings & & $17(34 \%)$ \\
Lobar Pneumonia & $19(38 \%)$ & $33(66 \%)$ \\
Bronchopneumonia & $31(62 \%)$ & 0 \\
$\quad$ Arterial Blood Gas & 1 & 1 \\
Respiratory Acidosis & 2 & \\
Respiratory Alkalosis & & \\
\hline
\end{tabular}

Table 2. Characteristics of Fever and cough in both the groups

\begin{tabular}{|c|c|c|c|c|}
\hline Parameters & & Group I & Group II & p value \\
\hline \multirow[t]{2}{*}{ Duration of fever (days) } & $<7$ & $38(76 \%)$ & $49(98 \%)$ & \multirow{2}{*}{0.001} \\
\hline & $7-15$ & $12(24 \%)$ & $1(2 \%)$ & \\
\hline \multirow[t]{2}{*}{ Intensity of fever } & High $\left(102^{\circ} \mathrm{F}\right)$ & $30(60 \%)$ & $32(64 \%)$ & \multirow{2}{*}{0.83} \\
\hline & $\operatorname{Low}\left(100^{\circ} \mathrm{F}-101^{\circ} \mathrm{F}\right)$ & $20(40 \%)$ & $18(36 \%)$ & \\
\hline \multirow[t]{2}{*}{ Character of fever } & Intermittent & $45(90 \%)$ & $46(92 \%)$ & \multirow{2}{*}{1.00} \\
\hline & Continuous & $5(10 \%)$ & $4(8 \%)$ & \\
\hline \multirow[t]{3}{*}{ Other fever parameters } & Chills & $3(6 \%)$ & $4(8 \%)$ & \multirow{3}{*}{1.00} \\
\hline & Rigors & $8(16 \%)$ & $8(16 \%)$ & \\
\hline & Diurnal Variation & $1(2 \%)$ & $0(0 \%)$ & \\
\hline \multirow[t]{2}{*}{ Duration of Cough } & $<7$ days & $37(74 \%)$ & $46(92 \%)$ & \multirow{2}{*}{0.03} \\
\hline & $7-15$ days & $13(26 \%)$ & $4(8 \%)$ & \\
\hline Productive cough & & $4(8 \%)$ & $2(4 \%)$ & 0.67 \\
\hline Diurnal Variation of cough & & $1(2 \%)$ & $0(0 \%)$ & 1.00 \\
\hline
\end{tabular}

Table 3. Outcome Analysis

\begin{tabular}{|c|c|c|c|c|}
\hline & $\begin{array}{l}\text { Oral Amoxicillin } \\
\text { Group (Mean } \pm \\
\text { SD) }\end{array}$ & $\begin{array}{l}\text { IV Ceftriaxone } \\
\text { Group (Mean } \\
\pm \text { SD) }\end{array}$ & $\begin{array}{l}\text { Mean Difference } \\
(95 \% \mathrm{CI})\end{array}$ & P value \\
\hline $\begin{array}{l}\text { Time to defervescence of fever } \\
\text { (days) }\end{array}$ & $3.7 \pm 0.9$ & $3.66 \pm 0.8$ & $0.04(-0.298$ to 0.378$)$ & 0.81 \\
\hline $\begin{array}{l}\text { Settlement of respiratory distress } \\
\text { (days) }\end{array}$ & $3.22 \pm 0.85$ & $3 \pm 0.67$ & $0.22(-0.084$ to 0.524$)$ & 0.19 \\
\hline Subsidence of Cough (days) & $11.68 \pm 0.92$ & $11.14 \pm 1.06$ & $0.54(0.146$ to 0.934$)$ & 0.74 \\
\hline Duration of hospital stay (days) & $6.02 \pm 1.11$ & $7.24 \pm 1.18$ & $1.22(0.765$ to 1.675$)$ & $<0.0001$ \\
\hline
\end{tabular}


IV ceftriaxone group. Use of a third-generation cephalosporin was not associated with any change in the outcomes as compared to oral amoxicillin (Table 3 ), except the duration of hospital stay $(p<0.0001)$ which was longer with parenteral treatment.

\section{Discussion}

In recent literature, studies have proved the effectiveness of higher antibiotics like cephalosporins and macrolides in the treatment of CAP in children. ${ }^{18,20,23,24,25,26}$ Although there are only a few studies that have proven the effectiveness of oral beta-lactams in uncomplicated CAP, there is still some discrepancy regarding their use. ${ }^{27,28,29}$ Our study proves that the efficacy of amoxicillin in managing uncomplicated CAP is similar to that of third-generation cephalosporins with no difference in the time to defervescence of fever, subsidence of cough or settlement of respiratory distress and only shorter duration of hospital stay in the amoxicillin group. Rehman et $\mathrm{al}^{26}$ conducted a similar study where the efficacy of treatment in terms of fever and respiratory rate after 24 hours follow up was analyzed. Amoxicillin was effective in $78.6 \%$ while IV ceftriaxone was effective in $83.4 \%$ children $(p=0.5)$. The mean hospital stay was 3.7 days in oral amoxicillin as compared to 4.1 days in IV ceftriaxone $(p=0.001)$. Pukander et $\mathrm{al}^{30}$ found that IV ceftriaxone was effective in $87 \%$ children while amoxicillin was effective in $80 \%$ children in CAP. Agweyu et al ${ }^{31}$ also demonstrated noninferiority of amoxicillin to benzylpenicillin for treatment of hospitalized children with severe pneumonia. Patel et $\mathrm{al}^{32}$, reported home based oral amoxicillin treatment was equivalent to hospital treatment for the first 48 hours in selected children and was less expensive than hospital treatment. Lodha et $\mathrm{al}^{25}$ in a systematic review examined antibiotics for community-acquired pneumonia in children and provided recommendations for countries with high case fatalities due to pneumonia. They recommended amoxicillin over co-trimoxazole in treatment of patients with CAP in ambulatory settings with co-amoxiclav and cefpodoxime as alternative second-line drugs. According to WHO Evidence Summaries $(2014)^{5}$, oral or injectable amoxicillin is the drug of choice in cases of pneumonia.

In our study, a step up to higher antibiotics was required in 3 patients. However, 2 of them had respiratory acid-base disturbances at the time of admission. This indicates that the children might have had compromised respiratory function at the time of admission. ${ }^{33}$ Hence, the reason for step-up can neither be attributed to failure of oral amoxicillin nor to the pre-existing resistance to beta-lactams. There is very less data available about the resistance of amoxicillin in organisms responsible for CAP. ${ }^{32}$ We didn't observe any clinical or statistical difference in both groups and both antibiotics seemed equally effective statistically and clinically. However, this doesn't mean that there is complete absence of resistance to beta-lactams in the community.

Our study demonstrates that oral amoxicillin is as effective as parenteral ceftriaxone for the treatment of uncomplicated CAP and reduces the number of days to hospitalization. Also, the side effects associated with parenteral therapy like injection site pain, extravasation are avoided. Use of effective lower class of antibiotics, spares the unnecessary abuse of the more broader spectrum drugs, thereby preventing anti-microbial resistance.

\section{Compliance with Ethical Standards}

Funding: None

Conflict of Interest: None

\section{References:}

1. Ayede AI, Kirolos A, Fowobaje KR, Williams LJ, Bakare AA, Oyewole OB, et al. A prospective validation study in SouthWest Nigeria on caregiver report of childhood pneumonia and antibiotic treatment using Demographic and Health Survey (DHS) and Multiple Indicator Cluster Survey (MICS) questions. J Glob Health. 2018;8:020806.

2. World Health Organization. Pneumonia fact sheet. Media centre. 2019. Available at URL: https://www.who.int/ news-room/fact-sheets/detail/pneumonia. Accessed on 28th February 2021.

3. Harris M, Clark J, Coote N, Fletcher P, Harnden A, McKean M, Thomson A; British Thoracic Society Standards of Care Committee. British Thoracic Society guidelines for the management of community acquired pneumonia in children: update 2011. Thorax. 2011;66 Suppl 2:ii1-23.

4. Lanks CW, Musani AI, Hsia DW. Community-acquired Pneumonia and Hospital-acquired Pneumonia. Med Clin North Am. 2019;103:487-501.

5. World Health Organization (WHO). Revised WHO classification and treatment of childhood pneumonia at health facilities: evidence summaries. 2014 Jun 11. Available at URL: https://apps.who.int/iris/bitstream/ handle/ 10665/137319/9789241507813_eng.pdf; jsessionid=B07BA086A2FA7B8BF00F69DBA149AE86? sequence $=1$. Accessed on 28th February 2021.

6. Mandell LA. Community-acquired pneumonia: An overview. Postgrad Med. 2015;127:607-615.

7. Ning $G$, Wang $X$, Wu $D$, Yin $Z$, Li $Y$, Wang $H$, Yang W. The etiology of community-acquired pneumonia among children under 5 years of age in mainland China, 20012015: A systematic review. Hum Vaccin Immunother. 2017; 13:2742-2750.

8. Çilli A, Sayıner A, Çelenk B, Şakar Coşkun A, Kılınç O, Hazar $A$, et al. Antibiotic treatment outcomes in communityacquired pneumonia. Turk J Med Sci. 2018;48:730-736.

9. François $P$, Desrumaux $A$, Cans $C$, Pin I, Pavese $P$, Labarère J. Prevalence and risk factors of suppurative complications in children with pneumonia. Acta Paediatr. 2010;99:861866.

10. Mathur S, Fuchs A, Bielicki J, Van Den Anker J, Sharland $M$. Antibiotic use for community-acquired pneumonia in neonates and children: WHO evidence review. Paediatr Int Child Health. 2018;38(sup1):S66-S75.

11. Nascimento-Carvalho AC, Nascimento-Carvalho CM. Clinical management of community-acquired pneumonia in young children. Expert Opin Pharmacother. 2019;20:435442.

12. le Roux DM, Zar HJ. Community-acquired pneumonia in children-a changing spectrum of disease. Pediatr Radiol. 2017;47:1392-1398.

13. Zhong NS, Sun T, Zhuo C, D'Souza G, Lee SH, Lan NH, et al. Ceftaroline fosamil versus ceftriaxone for the treatment 
of Asian patients with community-acquired pneumonia: a randomised, controlled, double-blind, phase 3, noninferiority with nested superiority trial. Lancet Infect Dis. 2015;15:161-171.

14. File TM Jr, Low DE, Eckburg PB, Talbot GH, Friedland HD, Lee $\mathrm{J}$, et al. Integrated analysis of FOCUS 1 and FOCUS 2: randomized, doubled-blinded, multicenter phase 3 trials of the efficacy and safety of ceftaroline fosamil versus ceftriaxone in patients with community-acquired pneumonia. Clin Infect Dis. 2010;51:1395-1405. Erratum in: Clin Infect Dis. 2011;52:967.

15. Cannavino CR, Nemeth A, Korczowski B, Bradley JS, O'Neal $T$, Jandourek A, et al. A Randomized, Prospective Study of Pediatric Patients With Community-acquired Pneumonia Treated With Ceftaroline Versus Ceftriaxone. Pediatr Infect Dis J. 2016;35:752-759.

16. Williams DJ, Hall M, Shah SS, Parikh K, Tyler A, Neuman MI, et al. Narrow vs broad-spectrum antimicrobial therapy for children hospitalized with pneumonia. Pediatrics. 2013;132: e1141-1148.

17. Eljaaly K, Wali H, Basilim A, Alharbi A, Asfour HZ. Clinical cure with ceftriaxone versus ceftaroline or ceftobiprole in the treatment of staphylococcal pneumonia: a systematic review and meta-analysis. Int J Antimicrob Agents. 2019;54:149-153.

18. Wang $\mathrm{CY}$, Chen YH, Fang C, Zhou MM, Xu HM, Jing CM, et al. Antibiotic resistance profiles and multidrug resistance patterns of Streptococcus pneumoniae in pediatrics: A multicenter retrospective study in mainland China. Medicine (Baltimore). 2019;98:e15942.

19. Meng D, Cao Y, Fu J, Chen R, Lu L, Tu Y. Sonographic assessment of ceftriaxone-associated biliary pseudolithiasis in Chinese children. J Int Med Res. 2010;38:2004-2010.

20. Leyenaar JK, Shieh MS, Lagu T, Pekow PS, Lindenauer PK. Comparative effectiveness of ceftriaxone in combination with a macrolide compared with ceftriaxone alone for pediatric patients hospitalized with community-acquired pneumonia. Pediatr Infect Dis J. 2014;33:387-392.

21. Williams DJ, Hall M, Shah SS, Parikh K, Tyler A, Neuman MI, et al. Narrow vs broad-spectrum antimicrobial therapy for children hospitalized with pneumonia. Pediatrics. 2013;132:e1141-e1148.

22. Thompson JW, Jacobs RF. Adverse effects of newer cephalosporins. An update. Drug Saf. 1993;9:132-142.

23. Blyth CC, Gerber JS. Macrolides in Children With Community-Acquired Pneumonia: Panacea or Placebo? J Pediatric Infect Dis Soc. 2018;7:71-77.

24. Leung AKC, Wong AHC, Hon KL. Community-Acquired Pneumonia in Children. Recent Pat Inflamm Allergy Drug Discov. 2018;12:136-144.

25. Lodha R, Kabra SK, Pandey RM. Antibiotics for communityacquired pneumonia in children. Cochrane Database Syst Rev. 2013;2013:CD004874.

26. Rehman Z, Shah IA. Comparison of efficacy of Amoxicillin versus Ceftriaxone in children with pneumonia. JSMC. 2018;7:127-130.

27. Wubbel L, Muniz L, Ahmed A, Trujillo M, Carubelli C, McCoig $C$, et al. Etiology and treatment of community-acquired pneumonia in ambulatory children. Pediatr Infect Dis J. 1999;18:98-104.

28. Principi N, Esposito S. Management of severe communityacquired pneumonia of children in developing and developed countries. Thorax. 2011;66:815-822.

29. Harris JA, Kolokathis A, Campbell M, Cassell GH, Hammerschlag MR. Safety and efficacy of azithromycin in the treatment of community-acquired pneumonia in children. Pediatr Infect Dis J. 1998;17:865-871.

30. Pukander JS et al. Intravenous ceftriaxone versus intravenous amoxicillin in the treatment of pediatric patient with pneumonia, Pediatric Infectious Journal p118-121

31. Agweyu A, Gathara D, Oliwa J, Muinga N, Edwards T, Allen $E$, et al. Oral amoxicillin versus benzyl penicillin for severe pneumonia among Kenyan children: a pragmatic randomized controlled noninferiority trial. Clin Infect Dis. 2015;60:1216-1224.

32. Patel AB, Bang A, Singh M, Dhande L, Chelliah LR, Malik A, et al. A randomized controlled trial of hospital versus home based therapy with oral amoxicillin for severe pneumonia in children aged 3 - 59 months: The IndiaCLEN Severe Pneumonia Oral Therapy (ISPOT) Study. BMC Pediatr. 2015;15:186.

33. Rennis DK, Krishnakumar EV. Role of initial Arterial Blood Gas variations in predicting the outcome of pneumonia patients with type I/II respiratory failure. International Journal of Advances in Medicine. 2016;3:313-318. 